\title{
Comparison of Visante and slit- lamp anterior segment optical coherence tomography in imaging the anterior chamber angle
}

${ }^{1}$ Singapore Eye Research Institute and Singapore National Eye Center, Singapore, Singapore

${ }^{2}$ Tan Tock Seng Hospital, Singapore, Singapore

${ }^{3}$ Zhongshan Ophthalmic Center, Guangzhou, China

${ }^{4}$ Yong Loo Lin School of Medicine, National University of Singapore, Singapore, Singapore

Correspondence: T Aung, Singapore Eye Research Institute, Singapore National Eye Center, 11 Third Hospital Avenue, Singapore 168751, Singapore Tel: + 654880775 ; Fax: + 654880775

E-mail: tin11@

pacific.net.sg

${ }^{5}$ These authors contributed equally to this work and are joint first authors

Received: 10 July 2008 Accepted in revised form: 11 April 2009 Published online: 12 June 2009

\section{Abstract}

Aims To compare the diagnostic performance of two anterior segment optical coherence tomography (AS-OCT) devices in assessing the anterior chamber angle (ACA). Methods Visante-OCT and slit-lamp-OCT (SL-OCT) were performed on 101 patients by a single operator. The AS-OCT images were processed by customised 'dewarping' software and assessed by two glaucoma specialists masked to clinical findings. A closed ACA was defined by the presence of contact between the iris and angle anterior to the scleral spur. Measurements of the ACA, anterior chamber depth (ACD), and pupil diameter were analysed. Gonioscopy was performed by another examiner masked to AS-OCT findings. Results Qualitative analysis could be carried out in $83(83 \%)$ eyes and quantitative analysis in $61(60 \%)$ eyes. A closed angle in at least one quadrant of the eye was observed in $\mathbf{3 0}$ eyes with gonioscopy; Visante-OCT imaging identified 29 of $30(97 \%)$ and SL-OCT imaging identified 27 of $30(90 \%)$ of these eyes $(P=0.50$, McNemar test). Visante-OCT detected more eyes with at least one closed quadrant than SLOCT (55 vs 46 eyes, respectively, $P=0.01$ ). Overall, SL-OCT had better agreement with gonioscopy than with Visante-OCT. Both ASOCTs showed good agreement for ACD measurements; however, SL-OCT tended to provide consistently higher ACA measurements and smaller pupil diameters than did Visante-OCT.

Conclusions Both AS-OCT devices detected most of the eyes with closed ACA on
LM Sakata ${ }^{1,5}$, TTL Wong ${ }^{1,5}$, H-T Wong ${ }^{2}$, RS Kumar' ${ }^{1}$, HM Htoon ${ }^{1}$, HT Aung ${ }^{1}, \mathrm{M} \mathrm{He}^{3}$ and T Aung ${ }^{1,4}$ gonioscopy. However, Visante-OCT detected more closed ACAs than did SL-OCT. The better agreement between SL-OCT and gonioscopy is likely because of the use of visible light during both examinations. The ACA measurements obtained with each device are not interchangeable.

Eye (2010) 24, 578-587; doi:10.1038/eye.2009.141; published online 12 June 2009

Keywords: angle closure glaucoma; angle closure; closed angle; optical coherence tomography

Introduction

Anterior segment optical coherence tomography (AS-OCT) is a new technology capable of obtaining real-time images of the anterior chamber angle (ACA), and represents a non-contact method for detecting eyes at risk for angle closure. ${ }^{1-6}$ Two commercial AS-OCT devices are currently available-Visante-OCT (Carl-Zeiss Meditec, Dublin, CA, USA) and slitlamp-OCT (SL-OCT, Heildelberg Engineering, Heildelberg, Germany). Although both devices rely on the principle of low-coherence interferometry, there are some differences in the way each device acquires and processes the images. Visante-OCT has an image acquisition speed 10 times faster than SL-OCT, and VisanteOCT images have a horizontal resolution of $60 \mu \mathrm{m}$ compared with $75 \mu \mathrm{m}$ for SL-OCT. During image acquisition, the gross positioning of the scanning beam with SL-OCT is performed by using a low-intensity narrow light beam from 
the slit-lamp, whereas for the Visante-OCT, gross positioning is performed automatically by the device after the examiner points at the centre of the pupil (with a mouse click) on the live video screen of the monitor. During image acquisition with SL-OCT, moving the superior and inferior eyelids out of the way represents a relatively easy task in most eyes, as the SL-OCT system is incorporated into a slit-lamp. On the customised platform of the Visante-OCT, moving the superior and inferior eyelids out of the way can be quite difficult, particularly for the left eye.

The cross-sectional anterior segment images obtained with both AS-OCT devices can be evaluated qualitatively or quantitatively. The qualitative analysis assesses the presence of irido-trabecular contact, whereas for the quantitative analysis, several anterior chamber parameters can be measured, including anterior chamber depth (ACD) and ACA parameters, such as angle opening distance (AOD) and trabecular-iris space area (TISA).

The aim of this study was to compare the diagnostic performance of Visante-OCT and SL-OCT in detecting closed ACAs using gonioscopy as the reference standard, and also to compare the quantitative parameters measured by these two devices.

\section{Methods}

In this hospital-based study, consecutive patients were recruited from a Glaucoma Clinic at a Singapore hospital from January to June 2007. Informed consent was obtained from all participants, and the study had the approval of the hospital's Institutional Review Board and adhered to the tenets of the Declaration of Helsinki.

After an interview about previous medical and ophthalmic history, each patient underwent the following examinations on the same day: visual acuity, slit-lamp biomicroscopy, imaging with the SL-OCT and Visante-OCT, and gonioscopy. Imaging was performed before any procedure that involved contact with the eye. Patients were excluded if they had a history of previous intraocular surgery or penetrating trauma, or any cornea opacities or abnormalities that precluded AS-OCT imaging (those who had previously undergone laser iridotomy were not excluded).

\section{Gonioscopy}

Gonioscopy was performed in the dark in all cases by a single examiner with glaucoma fellowship training (HTW) who was masked to AS-OCT findings. Static and dynamic gonioscopy was performed using a Sussman 4-mirror lens (Volk Optical Inc, Mentor, OH, USA), at high magnification $(\times 16)$, with the eye in the primary position of gaze. A 1-mm light beam was reduced to a very narrow slit, and care was taken to avoid light falling on the pupil and to avoid accidental indentation. Slight tilting of the gonioscopy lens was permitted in an attempt to gain a view over the convexity of the iris. The ACA in each quadrant was graded

using the Scheie grading system, which is based on anatomical structures observed during gonioscopy (grade I — visible ciliary body, grade II — visible scleral spur, grade III - visible anterior trabecular meshwork, grade IV - angle structures not visible). ${ }^{7}$

\section{Anterior segment optical coherence tomography}

Anterior segment imaging was performed using the commercially available Visante-OCT (model 1000, software version 1.0, Carl-Zeiss Meditec) and SL-OCT devices (SL-OCT software version 1.1, Heildelberg Engineering). The details of AS-OCT imaging technology have been described previously. ${ }^{8}$ Image acquisition using AS-OCT was performed under the same room lighting conditions, in random order by a single experienced operator (HTA) masked to other test results. Scans of the ACA of each eye were obtained at the 3 and 9 o'clock positions (horizontal), and at the 6 and 12 o'clock positions (vertical). As the eyelids may interfere with AS-OCT image acquisition of the ACA at 6 and 12 o' clock positions, the lower and upper lids were gently moved out of the way by the operator to image the inferior/ superior angle - taking care to avoid inadvertent pressure on the globe. After acquisition, the scanned images were processed using customised software ('dewarping' software), which compensates for index of refraction transition at the air-tear interface and for the different group indices in air, cornea, and aqueous to correct the physical dimensions of the images. ${ }^{9}$ Of note, a low-intensity narrow light beam from the slit-lamp was kept on during the SL-OCT scan acquisition to facilitate gross adjustment of the OCT scanning beam location, as the scanning beam scans structures along the same line as the slit-lamp beam.

\section{Qualitative analysis}

The open/closed ACA status in each of the four quadrants of the eye was assessed by two examiners with glaucoma subspecialty training (LMS and TA working together) masked to other test results. The ACA was considered 'closed' on AS-OCT imaging if any contact between the iris and angle wall anterior to the scleral spur was noted. On gonioscopy, an ACA quadrant was considered 'closed' if the posterior trabecular meshwork could not be seen in the primary position without indentation (Scheie grade 3 or 4 ). 


\section{Quantitative analysis}

The quantitative analysis on SL-OCT images were performed by the custom-built software included in the SL-OCT device, whereas for Visante-OCT images, the analysis was performed with a new analysis software (the Zhongshan Angle Analysis Program (ZAAP), Guangzhou, China). ${ }^{10}$ For images from both AS-OCT devices, two fellowship-trained glaucoma specialists (LMS and RSK working together), masked to the other test results, marked out the scleral spur location in nasal and temporal quadrants of each eye, after which both the analysis softwares automatically provided the quantitative analysis (Figure 1). The scleral spur was determined as the point at which there was a change in curvature of the inner surface of the angle wall, often appearing as an inward protrusion of the sclera. The following parameters were assessed (Figure 2):

(1) Angle opening distance at $500 \mu \mathrm{m}$ and $750 \mu \mathrm{m}$ anterior to the scleral spur $\left(\mathrm{AOD}_{500}\right.$ and $\mathrm{AOD}_{750}$, respectively) $)^{11,12}$

(2) Trabecular-iris space area at 500 and $750 \mu \mathrm{m}$ from the scleral spur TISA $_{500}$ and TISA $_{750}$, respectively). ${ }^{8}$

(3) Central ACD, measured along a line perpendicular to the anterior surface of the lens to the posterior surface of the cornea.

(4) Scleral spur to scleral spur distance, measured as the shortest distance between the two scleral spur points defined by the examiners.

(5) Pupil diameter, measured as the shortest distance between the pupil edges of the iris cross-sections

\section{Statistical analysis}

One eye per patient was randomly selected for analysis. Parametric and non-parametric tests were used to compare continuous variables, according to data distribution. The $\chi^{2}$-test was used to compare categorical data. The McNemar test was used to compare differences in the distribution of a categorical variable between two dependent samples. Kappa statistic was used to assess the agreement between categorical variables. However, the kappa statistic may be affected by trait prevalence (distribution) and base rates. ${ }^{13,14}$ In an attempt to deal with these well-known limitations, Gwet ${ }^{14}$ proposed using AC1 statistics to assess the agreement between raters in situations wherein the prevalence of positive classifications may lead to inconsistent results. AC1 results are interpreted in a similar way as kappa statistics. ${ }^{13,14}$ Bland-Altman analyses were performed to evaluate the agreement between the two devices, and the mean bias and the $95 \%$ limits of agreement for each comparison were calculated. ${ }^{15,16} \mathrm{~A} P$-value $<0.05$ was considered statistically significant. Statistical analyses were performed using JMP 5 (SAS Institute, Inc., Cary, NC, USA), and MedCalc (Mariakerke, Belgium) softwares.

\section{Results}

A total of 101 patients were recruited. The mean age of participants was $62.4 \pm 9.6$ years (range $41-89$ years). A total of $57(58 \%)$ patients were female, the majority were Chinese (88 (87\%)), the others being Malay (2 (2\%)), Indian $(7(7 \%))$, and other races $(4(4 \%))$. Patients included in the study represented consecutive patients attending a glaucoma clinic, and had a diagnosis of primary open-angle glaucoma, primary angle-closure glaucoma, or glaucoma suspect.

\section{Qualitative analysis}

Five patients had to be excluded from the analysis because of failure in obtaining SL-OCT images (the examiner was not able to move the eyelids out of the way to acquire the SL-OCT scan in four patients, and the SL-OCT software could not properly 'dewarp' the image in one patient). Images of 11 patients could not be graded because of poor image quality or poor definition of the scleral spur (three patients with SL-OCT imaging, two patients with Visante-OCT imaging, and six patients with both devices). There were two patients with incomplete gonioscopy results. Furthermore, qualitative analysis only considered 83 eyes ( $83 \%$ of total) in which the ACA status could be assessed in all four quadrants with both AS-OCT devices. Of note, there were no difference in the mean age, gender, or race distribution, or the mean Scheie grading between the 18 excluded cases and 83 included cases $(P>0.351$, data not shown).

\section{Gonioscopy vs AS-OCT}

A closed ACA in at least one quadrant of the eye was observed in 30 eyes with gonioscopy, in 55 eyes with Visante-OCT, and in 46 eyes with SL-OCT (Table 1). Using gonioscopy as the reference standard, VisanteOCT identified 29 of 30 (97\%) of the eyes with $\geqslant 1$ closed ACA on gonioscopy, whereas SL-OCT imaging detected $27(90 \%)$ of these eyes ( $P=0.500$, McNemar test)

(Table 2). Similar results were observed in eyes with a closed ACA in $\geqslant 2$ and $\geqslant 3$ quadrants on gonioscopy (Table 2). The agreement in detecting eyes with at least one closed ACA was higher between gonioscopy and SL-OCT than between gonioscopy and Visante-OCT (agreement in 61 of 83 eyes, $\mathrm{AC} 1=0.474$ vs agreement in 56 of 83 eyes, $\mathrm{AC} 1=0.350$, respectively) (Figure 3).

A total of 53 eyes had an open ACA in all the four quadrants of the eye on gonioscopy. However, Visante- 


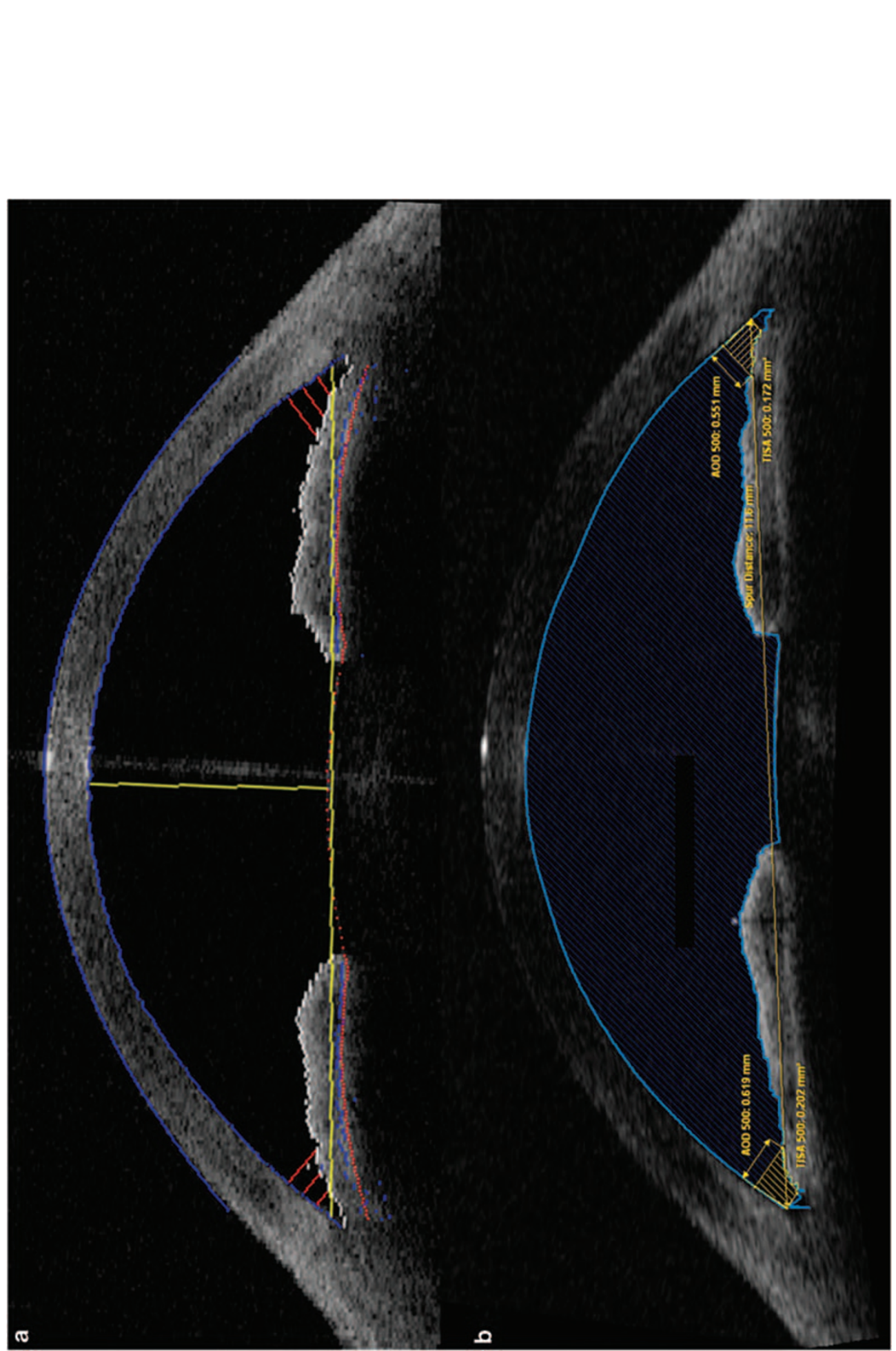

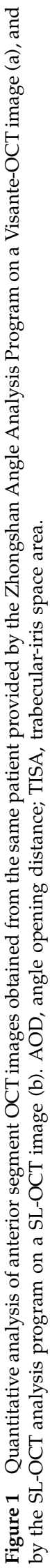


OCT detected at least one closed ACA quadrant in 26 (49\%) of these eyes, whereas SL-OCT detected at least one closed ACA quadrant in $19(36 \%)$ of these eyes ( $P=0.039$, McNemar test) (Table 2).

In the analysis by quadrants, both AS-OCT devices tended to detect more closed ACA quadrants than gonioscopy in the superior and inferior quadrants, but both AS-OCT devices tended to detect less or at least similar rates of closed ACA quadrants as compared with gonioscopy in the temporal and nasal quadrants (Table 3).

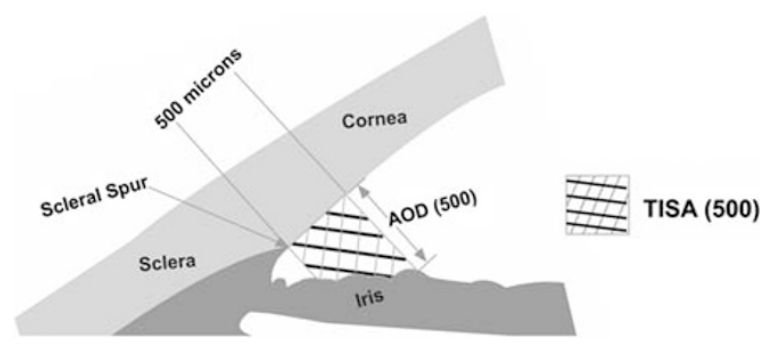

Figure 2 Diagrammatic representation of the ACA measurements: angle opening distance (AOD) and trabecular-iris space area at $500 \mu \mathrm{m}$ from the scleral spur. AOD, angle opening distance; TISA, trabecular-iris space area.

\section{Visante-OCT vs SL-OCT}

The agreement between Visante-OCT and SL-OCT was good (agreement in 72 of 83 eyes, $\mathrm{AC} 1=0.747$ ). When the two AS-OCTs were compared against each other, the Visante-OCT detected more eyes with closed ACA than the SL-OCT $(P=0.012$, McNemar test).

Visante-OCT also detected more closed ACA quadrants in total than did SL-OCT $(P<0.001$, McNemar Test) (Table 3). A closed ACA in Visante-OCT images was detected in $37(11 \%)$ quadrants in which SL-OCT images showed an open ACA, whereas a closed ACA in SL-OCT and an open ACA in Visante-OCT were found in only five quadrants (Figure 4). With both forms of AS-OCT imaging, the rates of closed ACA quadrants in the superior and inferior quadrants were significantly higher when compared with the nasal and temporal quadrants $\left(P<0.001, \chi^{2}\right.$-test $)$.

The intra-observer reproducibility in qualitatively grading AS-OCT images of all 83 eyes was good (kappa value for Visante-OCT 0.71 (95\% CI (confidence intervals) 0.63-0.78) and for SL-OCT 0.71 (95\% CI 0.63-0.79)). The intra-observer reproducibility in grading the ACA on gonioscopy as open or closed was performed in a separate subset of 20 eyes (not included in the study), and the kappa values ranged from 0.80 to 1.00 for the four quadrants.

Table 1 Distribution of closed anterior chamber angle by quadrants diagnosed by anterior segment optical coherence tomography and gonioscopy

\begin{tabular}{|c|c|c|c|}
\hline Closed quadrant (s) & Visante-OCT & $S L-O C T$ & Gonioscopy \\
\hline Total number of eyes & $55(100 \%)$ & $46(100 \%)$ & $30(100 \%)$ \\
\hline \multicolumn{4}{|l|}{ Eyes with one closed quadrant } \\
\hline Superior & $3(5 \%)$ & $6(13 \%)$ & $1(3 \%)$ \\
\hline Inferior & $8(14 \%)$ & $8(17)$ & $0(0 \%)$ \\
\hline Nasal & $1(2 \%)$ & $0(0 \%)$ & $1(3 \%)$ \\
\hline Temporal & $1(2 \%)$ & $1(2 \%)$ & $0(0 \%)$ \\
\hline \multicolumn{4}{|l|}{ Eyes with two closed quadrants } \\
\hline Superior/inferior & $18(33 \%)$ & $13(28 \%)$ & $4(14 \%)$ \\
\hline Superior/nasal & $1(2 \%)$ & $1(2 \%)$ & $1(3 \%)$ \\
\hline Superior/temporal & $1(2 \%)$ & $0(0 \%)$ & $2(7 \%)$ \\
\hline Inferior/nasal & $0(0 \%)$ & $0(0 \%)$ & $0(0 \%)$ \\
\hline Inferior/temporal & $0(0 \%)$ & $0(0 \%)$ & $0(0 \%)$ \\
\hline Nasal/temporal & $0(0 \%)$ & $0(0 \%)$ & $0(0 \%)$ \\
\hline \multicolumn{4}{|l|}{ Eyes with three closed quadrants } \\
\hline Superior/inferior/nasal & $8(14 \%)$ & $7(15 \%)$ & $1(3 \%)$ \\
\hline Superior/inferior/temporal & $1(2 \%)$ & $3(5 \%)$ & $1(3 \%)$ \\
\hline Inferior/temporal/nasal & $0(0 \%)$ & $0(0 \%)$ & $0(0 \%)$ \\
\hline Superior/temporal/nasal & $0(0 \%)$ & $1(2 \%)$ & $2(7 \%)$ \\
\hline \multicolumn{4}{|l|}{ Eyes with four closed quadrants } \\
\hline Superior/inferior/nasal/temporal & $13(24 \%)$ & $6(13 \%)$ & $17(57 \%)$ \\
\hline
\end{tabular}

OCT, optical coherence tomography; SL-OCT, slit-lamp optical coherence tomography. 
Table 2 Anterior segment optical coherence tomography results compared with gonioscopy findings in all 83 eyes

\begin{tabular}{|c|c|c|c|c|}
\hline & \multicolumn{4}{|c|}{ Gonioscopy } \\
\hline & $\begin{array}{l}\text { All quadrants } \\
\text { open }(\mathrm{n}=53)\end{array}$ & $\begin{array}{l}\text { At least one quadrant } \\
\text { closed }(\mathrm{n}=30)\end{array}$ & $\begin{array}{l}\text { At least two quadrants } \\
\text { closed }(\mathrm{n}=27)\end{array}$ & $\begin{array}{c}\text { At least three quadrants } \\
\text { closed }(\mathrm{n}=20)\end{array}$ \\
\hline \multicolumn{5}{|l|}{$S L-O C T$} \\
\hline All quadrants open & 34 & 3 & 2 & 2 \\
\hline One quadrant closed & 8 & 7 & 7 & 5 \\
\hline Two quadrants closed & 6 & 8 & 6 & 6 \\
\hline Three quadrants closed & 4 & 7 & 7 & 5 \\
\hline Four quadrants closed & 1 & 5 & 5 & 2 \\
\hline \multicolumn{5}{|l|}{ Visante-OCT } \\
\hline All quadrants open & 27 & 1 & 0 & 0 \\
\hline One quadrant closed & 7 & 6 & 5 & 4 \\
\hline Two quadrants closed & 13 & 7 & 6 & 4 \\
\hline Three quadrants closed & 3 & 6 & 6 & 4 \\
\hline Four quadrants closed & 3 & 10 & 10 & 8 \\
\hline
\end{tabular}

OCT, optical coherence tomography; SL-OCT, Slit-lamp optical coherence tomography.

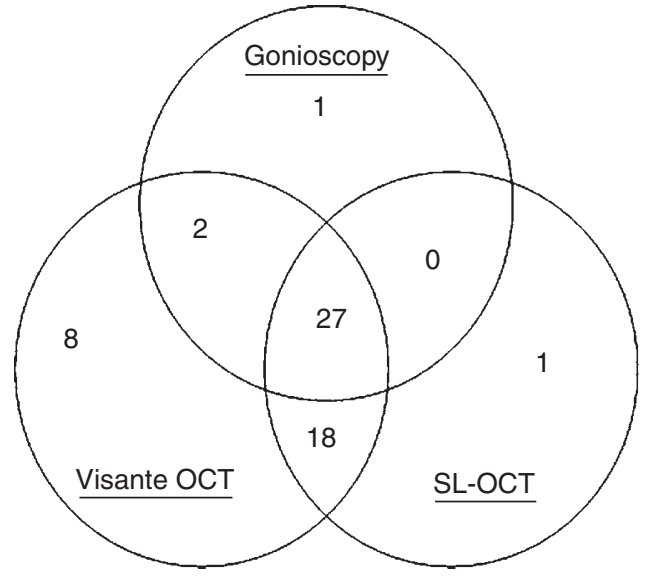

Figure 3 Venn diagram showing the agreement among gonioscopy, Visante-OCT, and slit-lamp OCT in detecting eyes with at least one quadrant with a closed angle.

\section{Quantitative analysis - Visante-OCT vs SL-OCT}

The exact location of the scleral spur was not detectable in either SL-OCT or Visante-OCT images from $40(40 \%)$ eyes (consisting 17 (17\%) eyes with SL-OCT, 15 (15\%) with Visante-OCT, and 8 (8\%) with both devices), and these patients were excluded from analysis. Further analysis only considered 61 eyes (60\% of total) in which both examiners agreed on the scleral spur location in both AS-OCT images. There were no difference in age, gender, race, or the mean angle width between the 40 excluded cases and 61 included cases $(P>0.354$, data not shown).

The ZAAP software was able to perform the quantitative analysis in all included 61 cases. However, SL-OCT software could not measure ACA parameters (both AOD and TISA) in two eyes, ACD in seven eyes, AOD measurements in three eyes, and TISA values in another nine eyes. In all these cases (total of 15 eyes), the SL-OCT software provided an error message ('not available'). Each of these eyes was excluded from the comparison analysis for the respective parameter. Of note, $10(67 \%)$ of these eyes had closed ACA in the SLOCT images.

When the measurements by Visante-OCT and SL-OCT were compared, the mean of differences (bias) was small for ACD measurements. However, the AOD and TISA parameters measured on SL-OCT images were significantly higher than the respective parameters measured by Visante-OCT (Table 4), and Bland-Altmann plots showed a consistent bias toward larger AOD and TISA values with the SL-OCT. On the other hand, the scleral spur to scleral spur distance measured on VisanteOCT were significantly higher than that measured by SLOCT (11.52 \pm 0.44 vs $11.41 \pm 0.40 \mathrm{~mm}, P<0.001$, respectively) (Table 4 ), and the pupil diameter measured on Visante-OCT was also significantly higher $(4.13 \pm 0.67$ vs $3.21 \pm 0.65 \mathrm{~mm}, P<0.001$, respectively)

\section{Discussion}

In the current study, both the Visante-OCT and the SL-OCT detected most of the eyes showing a closed angle on gonioscopy; however, both devices detected more eyes with closed ACA compared with gonioscopy. When the two AS-OCTs were compared against each other, the Visante-OCT detected more irido-trabecular contact than the SL-OCT, and the overall agreement between SL-OCT and gonioscopy was better than between Visante-OCT and gonioscopy. On quantitative analysis, the two 
Table 3 Number of quadrants with closed anterior chamber angles on gonioscopy, Visante-OCT and slit-lamp OCT in 332 quadrants of 83 eyes

\begin{tabular}{|c|c|c|c|c|}
\hline Quadrant & A-closed on gonioscopy & B-closed on Visante-OCT & C-closed on SL-OCT & $\mathrm{P}-$ value $e^{\mathrm{a}}$ \\
\hline Overall & $96(29 \%)$ & $132(40 \%)$ & $100(30 \%)$ & $\begin{array}{l}A \text { vs } B<0.001 \\
A \text { vs } C=0.758 \\
B \text { vs } C<0.001\end{array}$ \\
\hline Superior & $29(35 \%)$ & $45(54 \%)$ & $37(45 \%)$ & $\begin{array}{l}A \text { vs } B=0.003 \\
A \text { vs } C=0.152 \\
B \text { vs } C=0.021\end{array}$ \\
\hline Inferior & $23(28 \%)$ & $48(58 \%)$ & $37(45 \%)$ & $\begin{array}{l}A \text { vs } B<0.001 \\
A \text { vs } C=0.007 \\
B \text { vs } C=0.007\end{array}$ \\
\hline Nasal & $22(26 \%)$ & $23(28 \%)$ & $15(18 \%)$ & $\begin{array}{l}A \text { vs } B=0.999 \\
A \text { vs } C=0.180 \\
B \text { vs } C=0.008\end{array}$ \\
\hline Temporal & $22(26 \%)$ & $16(19 \%)$ & $11(13 \%)$ & $\begin{array}{l}A \text { vs } B=0.263 \\
A \text { vs } C=0.027 \\
B \text { vs } C=0.179\end{array}$ \\
\hline
\end{tabular}

OCT, optical coherence tomography; SL-OCT, slit-lamp optical coherence tomography.

${ }^{\mathrm{a} M c N e m a r}$ test.

instruments showed good range of limits of agreement for ACD measurements; however, the limits of agreement observed for the ACA parameters (AOD and TISA) may be considered clinically significant - between 88 and $100 \mu \mathrm{m}$ for $\mathrm{AOD}_{500}$ and between 47and $51 \mu \mathrm{m}^{2}$ for TISA $_{500}$, with a consistent bias of lower ACA measurements with Visante-OCT images. These discrepancies between the two AS-OCT devices may be explained by the different conditions during image acquisition with the two devices. A low-intensity narrow slit of light from the slit-lamp was kept on during the image acquisition with SL-OCT to facilitate the positioning of the scan beam, as this was suggested in the SL-OCT manual. The slit of light from the slit-lamp probably caused constriction of the pupils and opening of the ACA in some eyes. Evidence for the effect of light was supported by the smaller pupil diameter measured on SL-OCT images. Thus, the better agreement between SL-OCT and gonioscopy is likely because of the use of visible light during both examinations.

It is important to note that the SL-OCT scanning light uses infrared light and it works independently of the slitlamp illumination system. Thus, as with Visante-OCT, SL-OCT scans can be obtained in completely dark conditions. However, it is likely that this procedure would demand more time for the correct placement of the SL-OCT scan beam at the apex of the cornea, as the examiner would have to estimate where the slit-lamp beam would be located without turning the slit-lamp on (just by looking at the SL-OCT device monitor). An alternative method would be to turn the slit-lamp off after the gross positioning of the scanning beam at the apex of the cornea, although it would be necessary to wait for some seconds for complete scotopic mydriasis to take place before acquiring the image. Further studies should assess how quick and feasible it is to acquire SL-OCT images with the slit-lamp light turned off.

Both Visante-OCT and SL-OCT detected at least one closed ACA in $49 \%$ and $36 \%$ (respectively) of eyes that showed an open angle in all four quadrants of the eye on gonioscopy. These findings are similar to that observed in previous studies comparing AS-OCT and gonioscopy, and confirm that AS-OCT detects more closed ACAs than gonioscopy. ${ }^{1,17}$ Possible reasons accounting for the discrepancies between gonioscopy and AS-OCT have been proposed. ${ }^{1,17}$ Although AS-OCT uses infrared light and does not require contact with the eye, it is possible that inadvertent indentation during gonioscopy may have opened the angle, in spite of efforts to avoid accidental indentation. ${ }^{1,17}$ Similarly, the use of minimum amount of visible light to evaluate the ACA during gonioscopy may have led to a certain degree of pupil constriction and angle opening. ${ }^{1,17}$ Thus, it is possible that gonioscopy may be missing eyes with iridotrabecular contact. The level of irido-angle contact required to define a closed ACA was also different with each technique. ${ }^{1,17}$ Using AS-OCT, a closed ACA was defined as any contact between the iris and angle wall anterior to the scleral spur, but if this contact did not occur up to the level of the trabecular meshwork, the angle would have been graded as open on gonioscopy. Thus, it is also possible that AS-OCT imaging may be overdetecting eyes with closed ACA (false-positive cases).

In the analysis by quadrants, both AS-OCT imaging methods tended to detect more closed ACA than did 


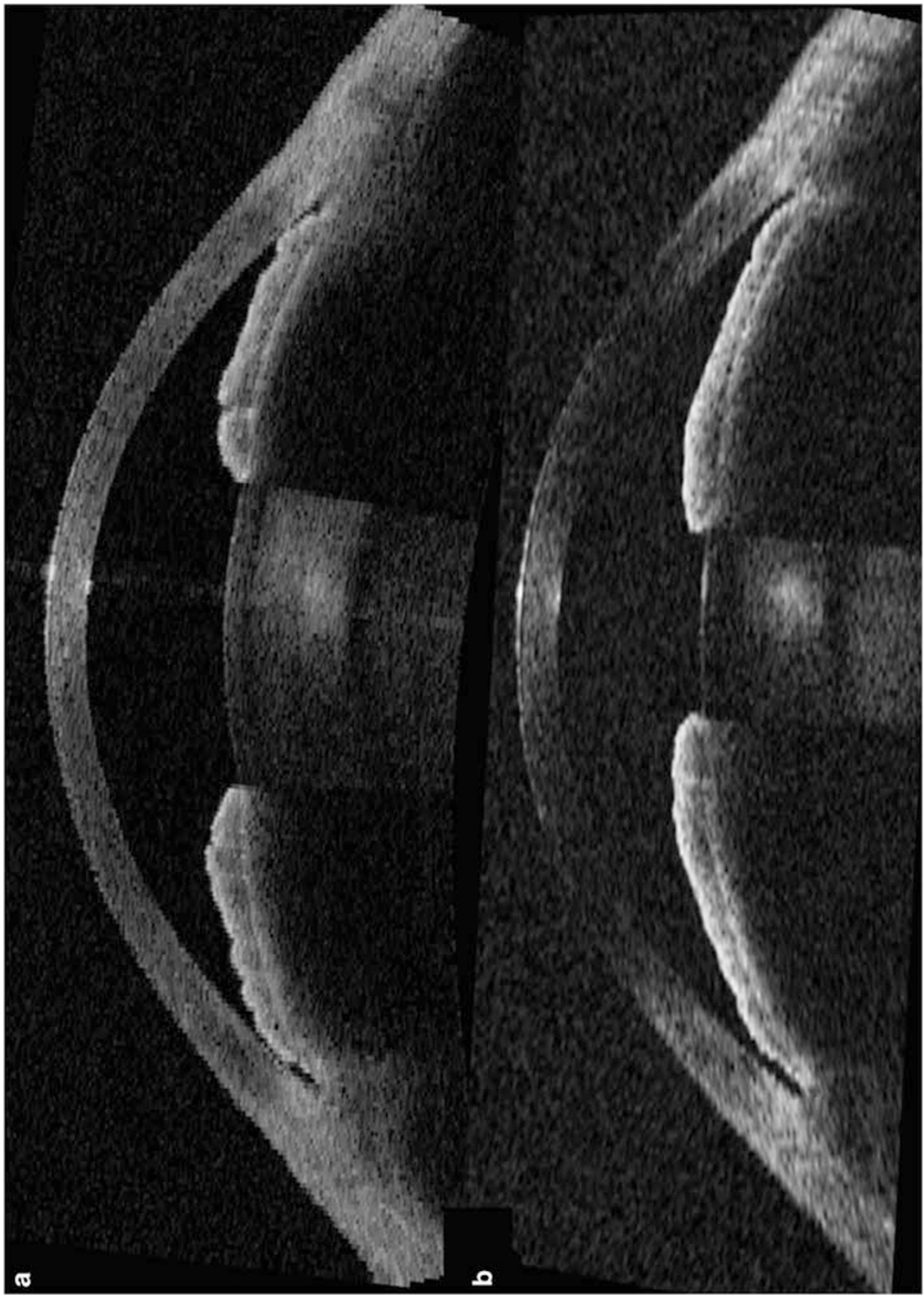

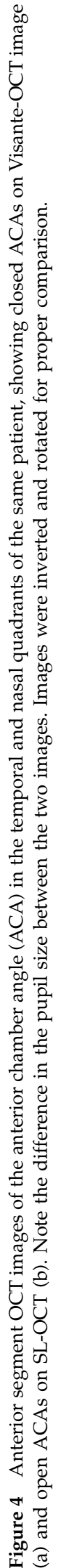


Table 4 Comparison of measurements obtained by the Visante-OCT and the slit lamp OCT

\begin{tabular}{lrcccc}
\hline & $\begin{array}{c}\text { SL-OCT } \\
\text { mean }(S D)\end{array}$ & $\begin{array}{c}\text { Visante-OCT } \\
\text { mean }(S D)\end{array}$ & $\begin{array}{c}\text { P-value } \\
\text { (two sided) }\end{array}$ & $\begin{array}{c}\text { Mean bias } \\
\text { (95\% CI) }\end{array}$ & $\begin{array}{c}\text { Limits of } \\
\text { Agreement }\end{array}$ \\
\hline ACD $(\mathrm{mm}, n=51)$ & $2.48(0.43)$ & $2.48(0.46)$ & 0.272 & $-0.006(-0.06$ to -0.00$)$ & -0.076 to 0.064 \\
SpurD $(\mathrm{mm}, n=61)$ & $11.41(0.40)$ & $11.52(0.44)$ & 0.001 & $-0.106(-0.05$ to -0.17$)$ & -0.567 to 0.350 \\
TAOD750 $(\mathrm{mm}, n=59)$ & $0.38(0.19)$ & $0.27(0.19)$ & $<0.001$ & $0.104(-0.12$ to 0.33$)$ & -0.070 to 0.280 \\
TAOD500 $(\mathrm{mm}, n=59)$ & $0.27(0.14)$ & $0.18(0.13)$ & $<0.001$ & $0.088(0.07$ to 0.12$)$ & -0.066 to 0.240 \\
NAOD750 $(\mathrm{mm}, n=56)$ & $0.37(0.19)$ & $0.26(0.17)$ & $<0.001$ & $0.110(0.08$ to 0.14$)$ & -0.100 to 0.320 \\
NAOD500 $\left(\mathrm{mm}^{2} n=56\right)$ & $0.28(0.15)$ & $0.17(0.12)$ & $<0.001$ & $0.110(0.08$ to 0.13$)$ & -0.070 to 0.290 \\
TTisa500 $\left(\mathrm{mm}^{2}, n=56\right)$ & $0.20(0.08)$ & $0.15(0.08)$ & $<0.001$ & $0.047(0.04$ to 0.06$)$ & -0.030 to 0.130 \\
NTisa500 $\left(\mathrm{mm}^{2}, n=52\right)$ & $0.18(0.09)$ & $0.14(0.08)$ & $<0.001$ & $0.051(0.04$ to 0.07$)$ & -0.050 to 0.150 \\
TTisa750 $\left(\mathrm{mm}^{2}, n=55\right)$ & $0.11(0.04)$ & $0.08(0.05)$ & $<0.001$ & $0.030(0.02$ to 0.04$)$ & -0.020 to 0.080 \\
NTisa750 $\left(\mathrm{mm}^{2}, n=52\right)$ & $0.11(0.05)$ & $0.07(0.04)$ & $<0.001$ & $0.030(0.02$ to 0.04$)$ & -0.030 to 0.100 \\
\hline
\end{tabular}

$\mathrm{ACD}$, anterior chamber depth; NAOD, nasal angle opening distance; NTisa, nasal trabecular-iris space area; OCT, optical coherence tomography; SL-OCT, slit-lamp optical coherence tomography; SpurD, scleral spur to scleral spur distance; TAOD, temporal angle opening distance; TTisa, temporal trabecular-iris space area.

${ }^{*} P$-values derived from paired $t$-test.

gonioscopy in the superior and inferior quadrants, but less or at least similar rates of closed ACA in the temporal and nasal quadrants. It is intriguing why this trend of higher rates of closed ACA in AS-OCT imaging compared with gonioscopy did not hold true in the temporal and nasal quadrants. This was observed in this study and has been previously reported. ${ }^{17}$ The manipulation of the eyelids to image the superior and inferior quadrants may have caused inadvertent narrowing of the angle. Of note, owing to the design of the Visante-OCT platform, moving the eyelids out of the way may be more difficult to do when using the VisanteOCT compared with SL-OCT, and this may have also influenced the observed results. Another possibility is that of grader bias for both gonioscopy and AS-OCT analyses. Interestingly, although a low-intensity narrow slit-beam of visible light was kept on during SL-OCT image acquisition, SL-OCT still detected more closed ACA than gonioscopy in the superior/inferior quadrants, but not in the temporal/nasal quadrants. These findings suggest that factors other than the influence of light may account for the discrepancies between these techniques among the quadrants. The influence of AS-OCT optical correction factors on ACA configuration within the four quadrants of the eye and also between individuals needs to be investigated.

Of note, the ACA of $16 \%$ of the eyes initially included in the study could not be qualitatively graded in at least one of the AS-OCT images. The inability to obtain and/or evaluate the AS-OCT images were due to technical difficulties in moving the eyelids out of the way and/or poor image quality, as well as difficulty in defining the scleral spur - likely due to the relatively poor transverse resolution of AS-OCT images, and/or anatomical variations between patients, or between the different quadrants of the eye. ${ }^{18}$ In fact, a problem we encountered when performing the quantitative analysis of the angle with both AS-OCT devices was that the exact location of the scleral spur could not be determined in approximately a quarter of OCT images (25\% with SL-OCT and 23\% with Visante-OCT).

Our study has some limitations. Although the examiner attempted to acquire cross-sectional images of the angle at 3,6,9, and 12 o'clock positions with both AS-OCTs, the exact location of these cross-sectional images obtained with each device may have varied. For quantitative analysis, it is possible that the scleral spur may not have been located at the same place in both Visante-OCT and SL-OCT images, thus affecting the comparison between measurements. The repeatability of the measured values in images obtained by the same operator and between different operators was not assessed, although this has been performed in other studies. ${ }^{11,19}$ To avoid corneal lesions that could interfere with the quality of images obtained with AS-OCT, gonioscopy was always performed after AS-OCT imaging. It is possible that this sequence of examinations may have influenced our results. The quantitative results reported in the current study are limited to the parameters measured on horizontal scans (nasal and temporal quadrants). Finally, it is not known whether there are inherent differences between the two different software programmes used for the measurements.

In summary, our study has shown that there are discrepancies between the rates of angle closure detected by gonioscopy and AS-OCT imaging, and between the two AS-OCT devices. It is unclear whether the higher rate of irido-trabecular contact detected by the VisanteOCT when compared with SL-OCT has any clinical relevance and further studies are required to determine the value of AS-OCT findings in the management of patients with angle closure. However, it is important to 
emphasise that the discrepancies in angle closure rates and ACA measurements by both AS-OCT devices should be taken into consideration when interpreting the results of these devices and that such measurements are not interchangeable.

\section{Acknowledgements}

Carl Zeiss Meditec and Heidelberg Engineering loaned the respective anterior segment OCTs and provided technical support. Dr Aung has received research support and honoraria for travel to conferences from Carl Zeiss Meditec. Dr HT Wong has received financial support and honoraria for travel to conferences from Carl Zeiss Meditec and Heidelberg Engineering.

\section{References}

1 Nolan WP, See JL, Chew PT, Friedman DS, Smith SD, Radhakrishnan $\mathrm{S}$ et al. Detection of primary angle closure using anterior segment optical coherence tomography in Asian eyes. Ophthalmology 2007; 114: 873-875.

2 Radhakrishnan S, Rollins AM, Roth JE, Yazdanfar S, Westphal V, Bardenstein DS et al. Real-time optical coherence tomography of the anterior segment at $1310 \mathrm{~nm}$. Arch Ophthalmol 2001; 119: 1179-1185.

3 Radhakrishnan S, Goldsmith J, Huang D, Westphal V, Dueker DK, Rollins AM et al. Comparison of optical coherence tomography and ultrasound biomicroscopy for detection of narrow anterior chamber angles. Arch Ophthalmol 2005; 123: 1053-1059.

4 Radhakrishnan S, Huang D, Smith SD. Optical coherence tomography imaging of the anterior chamber angle. Ophthalmol Clin North Am 2005; 18: 375-381, vi.

5 Izatt JA, Hee MR, Swanson EA, Lin CP, Huang D, Schuman JS et al. Micrometer-scale resolution imaging of the anterior eye in vivo with optical coherence tomography. Arch Ophthalmol 1994; 112: 1584-1589.

6 Konstantopoulos A, Hossain P, Anderson DF. Recent advances in ophthalmic anterior segment imaging: a new era of ophthalmic diagnosis? Br J Ophthalmol 2007; 91(4): 551-557.
7 Scheie HG. Width and pigmentation of the angle of the anterior chamber. A system of grading by gonioscopy. Arch Ophthalmol 1957; 58: 510-512.

8 Radhakrishnan S, Goldsmith J, Huang D, Westphal V, Dueker DK, Rollins AM et al. Comparison of optical coherence tomography and ultrasound biomicroscopy for detection of narrow anterior chamber angles. Arch Ophthalmol 2005; 123: 1053-1059.

9 Goldsmith JA, Li Y, Chalita MR, Westphal V, Patil CA, Rollins AM et al. Anterior chamber width measurement by high-speed optical coherence tomography. Ophthalmology 2005; 112: 238-244.

10 Pavlin CJ, Harasiewicz K, Foster FS. Ultrasound biomicroscopy of anterior segment structures in normal and glaucomatous eyes. Am J Ophthalmol 1992; 113: 381-389.

11 Console JW, Sakata LM, Aung T, Friedman DS, He M. Quantitative analysis of anterior segment optical coherence tomography images: the Zhongshan Angle Assessment Program. Br J Ophthalmol 2008; 92: 1612-1616.

12 Ishikawa H, Liebmann JM, Ritch R. Quantitative assessment of the anterior segment using ultrasound biomicroscopy. Curr Opin Ophthalmol 2000; 11: 133-139.

13 Chan YH. Biostatistics 104: correlational analysis. Singapore Med J 2003; 44: 614-619.

14 Inter-Rater Reliability: Dependency on Trait Prevalence and Marginal Homogeneity. Inter-Rater Reliability. Available at:http://www.stataxis.com/interrater.htm. Accessed 15 November 2006.

15 Bland JM, Altman DG. Statistical methods for assessing agreement between two methods of clinical measurement. Lancet 1986; 1(8476): 307-310.

16 Bland JM, Altman DG. Measuring agreement in method comparison studies. Stat Methods Med Res 1999; 8: 135-160.

17 Sakata LM, Lavanya R, Friedman DS, Aung HT, Gao H, Kumar RS et al. Comparison of gonioscopy and anteriorsegment ocular coherence tomography in detecting angle closure in different quadrants of the anterior chamber angle. Ophthalmology 2008; 115(5): 769-774.

18 Sakata LM, Lavanya R, Friedman DS, Aung HT, Seah SK, Foster PJ et al. Assessment of the scleral Spur in Visante anterior-segment ocular coherence tomography images. Arch Ophthalmol 2008; 126: 181-185.

19 Müller M, Dahmen G, Pörksen E, Geerling G, Laqua H, Ziegler A et al. Anterior chamber angle measurement with optical coherence tomography: intraobserver and interobserver variability. J Cataract Refract Surg 2006; 32(11): 1803-1808. 